\title{
DEVELOPMENT OF METHODS OF OPTIMIZATION AND PROMOTION OF WEB SITE IN SEARCH SYSTEMS
}

\author{
Olexandr Pushkar ${ }^{1}$, Yevhen Hrabovskyi ${ }^{2}$ \\ ${ }^{1}$ Department of Computer Systems and Technologies \\ Simon Kuznets Kharkiv National University of Economics, Kharkiv, Ukraine \\ ORCID: http://orcid.org/0000-0003-3592-3684 \\ ${ }^{2}$ Department of Computer Systems and Technologies \\ Simon Kuznets Kharkiv National University of Economics, Kharkiv, Ukraine \\ maxmin903@ukr.net
}

ARTICLE INFO

Article history:

Received date 04.12.2019

Accepted date 24.12.2019

Published date 31.01 .2020

Section:

Content Management Systems

D O I

$10.21303 / 2313-8416.2020 .001155$

\section{KE Y W OR D S}

\section{optimization}

website promotion

search engines

web server

SEO

processor

\section{A B S T R A C T}

The article analyzes the general features of the implementation of SEO optimization, which allows to determine the main aspects of website promotion in search engines. It is indicated that the key goal of SEO should be to increase the position of the site in the ranking of search engines, as well as to increase the rate of conversion of users to regular customers. Separately, the article analyzes unethical optimization methods - black and gray types of SEO, which are used for web resources that are not designed for long-term existence. A study of the basic steps is done to configure HTTPS for the site, which can be used in the process of improving the operation of the web server. This allows to optimize the main and additional processor resources. Two methods of reducing the number of processor operations performed for each client are considered, which are the use of persistent connections, which allow processing several requests within one connection at once, and the reuse of SSL session parameters. The key stages of the methodology for optimizing and promoting the site in search engines have been developed and analyzed, accounting for which ensures increased site efficiency and get a configured and optimized configuration file. The proposed technique is implemented using the Nginx web server as an example, which uses a model with a fixed number of processes.

(C) The Author(s) 2020. This is an open access article under the CC BY license http://creativecommons.org/licenses/by/4.0).

\section{Introduction}

The site is one of the components of a complex system of marketing activities of an organization to promote its brand and subject of activity. For the user to go to the desired web portal, search engine promotion is used-SEO. This tool is a set of measures to increase the position of the site in the search results of search engines. SEO, along with some other Internet marketing technologies, such as SMM (Social Media Marketing - promotion in social networks) and SEA (Search Engine Advertising - search advertising), are often used together in a system of events to promote an organization.

Being a fairly effective means of promotion, combined with the use of search engine optimization technology, the site is a powerful component of the marketing direction of the company. If to consider that a web resource in itself is a means of promotion, then SEO can be considered not just separately as a technology for promotion, but also as a technology for improving the effectiveness of a site.

In these conditions, today it is very urgent to develop a methodology for optimizing and promoting a site in search engines.

\section{Literature review}

The study [1] considers specific features of the use of multimedia components in terms of the process of website promotion. In [2], a diagnostic method for assessing the quality of SEO results is proposed. The scientific work [3] suggests ways to increase the effectiveness of SEO optimization art. The issues of website content optimization are considered in [4]. The main aspects of using the results of SEO optimization are systematized in the study [5]. The scientific work [6] highlights the use of various types of color models in terms of website promotion. In [7], the main aspects of optimizing mobile versions of sites are considered. The scientific work [8] describes a 
model of an electronic system interface that can be used to improve the site optimization process. The experience of promoting sites of various types is accumulated in [9]. The main aspects of the development of site optimization systems are given in the study [10].

So, as a result of the analysis of specialized literature, it is possible to conclude that there is no scientifically based methodology for optimizing and promoting the site in search engines.

\section{The aim and objectives of research}

The aim of research is development of methods for optimizing and promoting a site in search engines.

To achieve the aim, the following objectives are set:

- analysis of the general features of the implementation of SEO optimization;

- study of the basic steps to configure HTTPS for a website;

- development and analysis of key stages of the optimization and website promotion methodology in search engines.

\section{General features of SEO optimization implementation}

The goal of SEO, as follows from the definition, is the correct search engine promotion of the site (increasing the position of the site in the ranking of search engines), as well as increasing the rate of conversion of users to customers. Achieving this goal determines the performance of such search engine optimization tasks as:

1) creation of active inducing texts;

2) compliance with the rules for the preparation of texts for sites;

3) text optimization for search engines.

Speaking about the functions that search engine optimization performs, the following should be highlighted:

1) search promotion;

2) increasing popularity of the site;

3) increasing citation site;

4) conversion of visitors into customers.

To promote sites, it is possible to use two main types of SEO: white and black. From the name it is clear that the so-called "right" methods for the implementation of search engine optimization belong to whites. They are not prohibited by the rules of a particular search engine and do not deceive users. The opposite view is black SEO. This type involves achieving the goal at all costs, and is usually prohibited by search engines.

Black SEO includes methods:

1) cloaking - bots and site visitors see different content;

2) regulatory or search spam;

3) use of text invisible to Internet users, search engines see;

4) use of doorway pages for driving traffic.

The apparent ease of black search engine optimization often deceives those who want to use it, since the effect of using this type is short-lived. To maintain position, it is necessary to use high-quality, proven and correct SEO methods, that is, those related to white optimization.

In addition, there is a third type of SEO - gray, which is not at all a combination of the two described above. Gray is distinguished from black SEO only by the fact that there will be no specific sanctions on its application.

Despite the fact that the algorithms of search engines are regularly complicated, and search engine optimization as a whole turns into a site development under the rules of search engines, it is possible to find examples of using unethical SEO methods. Most often, black and gray SEO are used for web resources that are not designed for long-term existence: the likelihood of a ban is not critical if the goal of collecting fast traffic is achieved.

\section{Research results and their discussion}

The technique of optimizing and promoting a site in search engines is developed by the authors using the example of the Nginx web server. 
The first stage of the methodology for optimizing and promoting a site in search engines is setting up HTTPS for the site. Search engines require the use of a certificate and rank the site for the better, if the certificate is installed. So, the HTTPS configuration chain consists of several steps.

The first step in configuring HTTPS is to optimize HTTPS server. SSL operations consume additional processor resources. On multiprocessor systems, several work processes should be launched, no less than the number of processor cores available. There are two ways to reduce the number of these operations performed for each client: use of keepalive connections, which allow to process several requests within one connection, and reuse of SSL session parameters to prevent SSL handshake for concurrent and step-by-step messages. An example of a configuration optimized for multi-core systems with a 10-megabyte distribution session cache (Fig. 1).

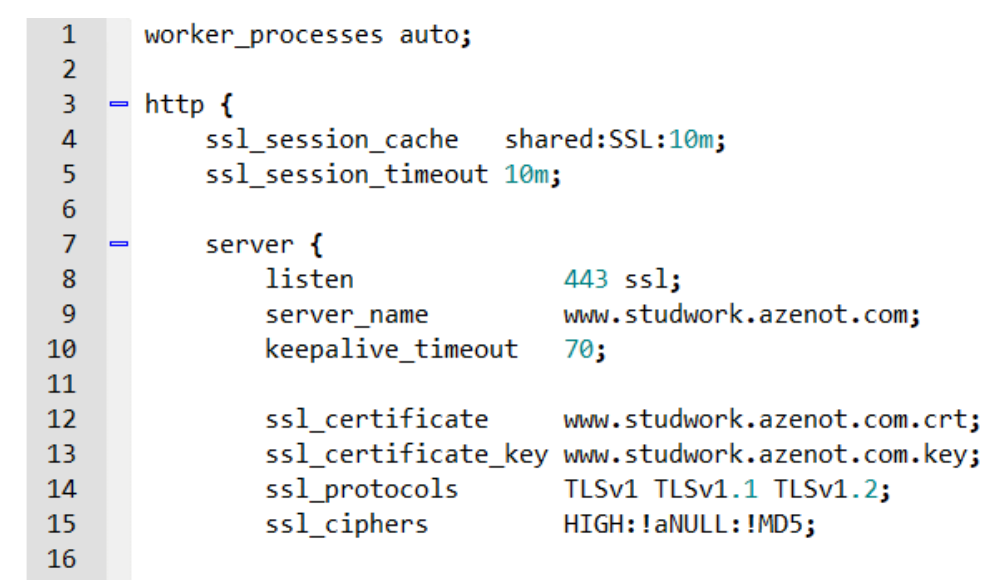

Fig. 1. «nginx.conf» configuration for a multi-core system

The second step in configuring HTTPS is the SSL certificate chain. Some browsers may issue warnings about a certificate signed by a well-known certification authority, while other browsers accept the same certificate without problems. This is because the issuing center signed it with an intermediate certificate, which is not in the certificate database of well-known trusted certification authorities, which is distributed with the browser. In this case, the certification authority provides a "chain" of certificates that should be attached to the server certificate. The server certificate should be placed in front of the certificate chain in a combination file. The resulting file should be specified in the ssl_certificate directive (Fig. 2).

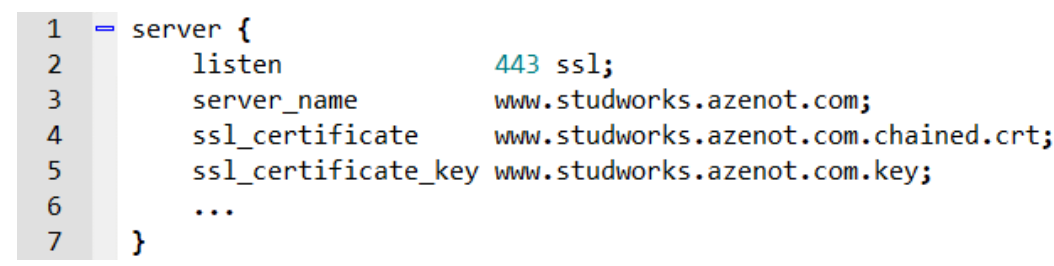

Fig. 2. Setting the ssl_certificate directive

It is possible to make sure that the server sends the full chain of certificates using the openssl command-line utility (Fig. 3). The output line about the site's certificate contains full information about the serial number, certificate repository, certification authority and its physical location.

The third step in configuring HTTPS is to combine the HTTP and HTTPS server. It is possible to configure a single server that serves both HTTP and HTTPS requests (Fig. 4).

The fourth step in configuring HTTPS is to establish an SSL certificate with multiple names. To do this, it is better to place information about the certificate file with several names in the file with its secret key at the HTTP configuration level so that all servers inherit their only copy of memory (Fig. 5).

The fifth step in configuring HTTPS is to provide a server name. A more general solution for running multiple HTTPS servers on the same IP address is the Server Name Indication exten- 
sion of the TLS protocol (SNI, RFC 6066), which allows the browser to pass the server name during SSL handshake.



Fig. 3. Running the openssl utility command



Fig. 4. HTTP/HTTPS configuration example

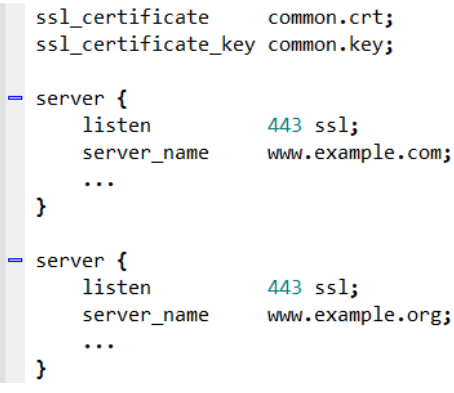

Fig. 5. Configuration of the file "nginx.conf" with two server directives

The second stage of the methodology for optimizing and promoting a site in search engines is setting up the Nginx web server to increase site performance. The faster the site's page is transferred to the client, the better it will affect search ranking, behavioral factors, performance rating, etc.

Nginx uses a model with a fixed number of processes that most effectively draws on available system resources:

1) single master process performs operations that require elevated rights, such as reading configurations and opening ports, and then creates a small number of child processes;

2) cache loader starts at startup to load the cache data that is located on the disk into RAM, and then terminates. Its work is designed so as not to consume a lot of resources;

3) cache manager wakes up periodically and removes cache objects from the hard drive in order to maintain its volume within the specified limit;

4) work processes do all the work. They process network connections, read data from disk, and write to disk in contact with backend servers.

Nginx documentation recommends that in most cases, to configure the number of workflows so that it equals the number of processor cores, which allows to use system resources as 
efficiently as possible. This mode must be set using the «worker_processes auto» directive in the «nginx.conf» configuration file.

When Nginx is under load, the work processes are mostly busy. Each of them processes many connections continuously, minimizing the number of context switches.

Each workflow is single-threaded and runs independently, accepting new connections and processing them. Processes interact with each other using shared memory for cache data, sessions, and other shared resources.

Relying on the basic principles of the Nginx web server and its configuration, it is possible to develop the following optimization methodology plan to achieve the best performance results:

1) request processing settings;

2) client-server connection settings;

3) caching file information;

4) logging the server output for further analysis (must be used to further optimize performance);

5) use of file compression to reduce their size and reduce the time it takes to give it to the client;

6) setting the quantity and quality of customer processing.

As a result of the implementation of the web server optimization method, Nginx should exit the configured and optimized configuration file

\section{Conclusions}

1. The analysis of the general features of SEO optimization implementation is done, which allows to determine the main aspects of website promotion in search engines.

2. A study of the basic steps to configure HTTPS for the site is done, which can be used in the process of improving the operation of the web server. This allows to optimize the main and additional processor resources.

3. The key stages of the methodology for optimizing and promoting the site in search engines are developed and analyzed. Their accounting provides an increase in the effectiveness of the site and getting a configured and optimized configuration file.

\section{References}

[1] Hrabovskyi, Y. (2019). Analysis of the use of multimedia components in modern mobile learning technologies. ScienceRise, 4 (57), 46-50. doi: http://doi.org/10.15587/2313-8416.2019.164597

[2] Hrabovskyi, Y. (2015). Methods of Assessment and Diagnosis of the Quality of Knowledge in E-Learning. Journal of Communication and Computer, 12 (6), 286-296. doi: http://oi.org/10.17265/1548-7709/2015.06.002

[3] Enge, E. (2015). The Art of SEO: Mastering Search Engine Optimization. O'reilly Media, 994.

[4] Norris, D. (2017). Content Machine: Use Content Marketing to Build a 7-Figure Business With Zero Advertising. Kindle Edition, 164.

[5] Patru, M. (2016). Making sense of MOOCs: A guide of policy-makers in developing countries. Paris : United Nations Educational, Scientific and Cultural Organization (UNESCO) and Commonwealth of Learning (COL), 102.

[6] Mohammadzadeh, D. M. (2012). Models of Colour Semiotics. Leeds: University of Leeds, 209.

[7] Tremblay, E. (2010). Educating the Mobile Generation - using personal cell phones as audience response systems in postsecondary science teaching. Journal of Computers in Mathematics and Science Teaching, 29 (2), 217-227.

[8] Hrabovskyi, Y., Fedorchenko, V. (2019). Development of the optimization model of the interface of multimedia edition. EUREKA: Physics and Engineering, 3, 3-12. doi: http://doi.org/10.21303/2461-4262.2019.00902

[9] Sursock, A. (2015). Trends 2015: Learning and Teaching in European Universities. Brussels: EUA, 128.

[10] Canessa, E., Zennaro, M. (2012). A Mobile Science Index for Development. International Journal of Interactive Mobile Technologies, 6 (1), 4-6. doi: http://doi.org/10.3991/ijim.v6i1.1751 